\title{
Continuous Time Analysis of the Response of the Pseudomedian and Related Filters to Periodic Signals
}

\author{
Mark A. Schulze and John A. Pearce \\ Department of Electrical and Computer Engineering and Biomedical Engineering Program \\ The University of Texas at Austin \\ Austin, Texas 78712
}

\begin{abstract}
We introduce a continuous time method to analyze the response of median, pseudomedian, average (mean), and midrange filters to certain periodic signals. The filter definitions are generalized to continuous time, and these definitions are applied to periodic signals such as triangle, square, and sinusoidal waves of varying frequencies. These operations yield "amplitude response" measures which are analytic functions of the frequency of the input signal. In addition, a "correlation" measure is defined to indicate the level of distortion introduced by each filter. Examples of this analysis for the median, pseudomedian, average, and midrange filters show similarities and differences among them.

Although these theoretical measures do not perfectly demonstrate the performance of the discrete time filters, continuous time analysis does provide valuable insights into the filter behavior. The response of the continuous time median filter shows its susceptibility to high frequency periodic noise and proves, again, the existence of infinite-length bi-valued fast-fluctuating roots of this filter. The pseudomedian filter, in contrast, completely attenuates amplitude-symmetric periodic signals above a certain frequency, and has no infinite-length fast-fluctuating roots. Continuous time filter analogues are therefore an important theoretical tool for understanding the behavior of both linear and nonlinear filters.
\end{abstract}

\section{INTRODUCTION}

Most image and signal processing filters are defined in discrete time; that is, they operate on a finite number of samples within a finite-sized window. However, as the number of samples in the filter window increases to infinity, some patterns in the behavior of the filters become evident. The behavior of filters acting on periodic signals is especially interesting. This paper develops the continuous time definitions for the median, pseudomedian, average (mean), and midrange filters and shows their responses to a variety of periodic signals. Although the pseudomedian filter was developed to mimic the median filter, in many ways its response to periodic signals resembles that of the midrange filter more closely, while the response of the median filter resembles that of the average filter more closely. The "amplitude response" of a continuous time filter is simply taken to be the peak response of the filter as a percentage of the peak amplitude of the input periodic signal. This is not equivalent to the amplitude response as defined by linear analysis, but linear techniques are not applicable to nonlinear filters. To further clarify the differences among the filters, a correlation measure is defined to determine the amount of distortion induced by filtering. It is impossible to draw specific conclusions about the behavior of digital filters from the continous time analysis, but comparing results for discrete and continuous time reveals significant similarities.

Copyright 1992 Society of Photo-Optical Instrumentation Engineers.

This paper was published in E. R. Dougherty, J. Astola, and C. G. Boncelet, Jr., eds., Nonlinear Image Processing III, Proc. SPIE, v. 1658, pp. 177-188, and is made available as an electronic reprint with permission of SPIE. One print or electronic copy may be made for personal use only. Systematic or multiple reproduction, distribution to multiple locations via electronic or other means, duplication of any material in this paper for a fee or for commercial purposes, or modification of the content of the paper are prohibited. 


\section{CONTINUOUS TIME FILTER DEFINITIONS}

\subsection{Pseudomedian Filter}

The pseudomedian and median filters are nonlinear techniques which are noted for their ability to preserve edges and reduce impulse noise in signals and images. The pseudomedian filter, introduced in 1985 by Pratt, Cooper, and Kabir ${ }^{1}$, is based upon concepts from mathematical morphology. It is defined as the average of the maximum of the minima of a set of subsequences and the minimum of the maximum of the same set of subsequences. The subsequences considered in a one-dimensional signal, for a filter window size of $2 \mathrm{~N}+1$, are all the contiguous subsequences of size $\mathrm{N}+1$. For example, the pseudomedian of a signal with values of $\{a, b, c, d, e\}$ inside a filter window of size five is:

$$
\begin{aligned}
\text { PMED }\{\mathrm{a}, \mathrm{b}, \mathrm{c}, \mathrm{d}, \mathrm{e}\} & =0.5 \cdot \max \{\min (\mathrm{a}, \mathrm{b}, \mathrm{c}), \min (\mathrm{b}, \mathrm{c}, \mathrm{d}), \min (\mathrm{c}, \mathrm{d}, \mathrm{e})\} \\
& +0.5 \cdot \min \{\max (\mathrm{a}, \mathrm{b}, \mathrm{c}), \max (\mathrm{b}, \mathrm{c}, \mathrm{d}), \max (\mathrm{c}, \mathrm{d}, \mathrm{e})\}
\end{aligned}
$$

Pratt $^{2}$ defines the operators maximin and minimax, which are exactly the two "halves" of the pseudomedian filter:

$$
\operatorname{PMED}\{\mathrm{a}, \mathrm{b}, \mathrm{c}, \mathrm{d}, \mathrm{e}\}=0.5 \bullet \operatorname{maximin}\{\mathrm{a}, \mathrm{b}, \mathrm{c}, \mathrm{d}, \mathrm{e}\}+0.5 \bullet \operatorname{minimax}\{\mathrm{a}, \mathrm{b}, \mathrm{c}, \mathrm{d}, \mathrm{e}\}
$$

The connection of the pseudomedian filter to mathematical morphology is clarified by this simplification, since the maximin is equivalent to the grayscale morphological operator open, and the minimax is equivalent to the close operator. The pseudomedian filter is thus the average of the morphological open and close. This result is demonstrated in more detail and for two dimensions by Schulze and Pearce ${ }^{3}$.

Extension of the definition of the pseudomedian filter to continuous time signals may be achieved directly from the morphological definition, or by simply converting the discrete maximum and minimum operators to the continuous supremum and infimum operators and considering all subsets of length one-half the window length inside the window. This gives the following expression for the continuous time pseudomedian, $\mathrm{PMED}_{\mathrm{ct}}$, of an input signal $f(t)$ at time $t=t_{0}$ with window size $w$.

$$
\begin{aligned}
\operatorname{PMED}_{\mathrm{ct}}\left\{\mathrm{f}\left(\left[\mathrm{t}_{\mathrm{o}}-\mathrm{w} / 2, \mathrm{t}_{\mathrm{o}}+\mathrm{w} / 2\right]\right)\right\} & =0.5 \bullet \sup \left(\left\{\inf \left(\mathrm{f}\left(\left[\mathrm{t}_{\mathrm{o}}+\beta-\mathrm{w} / 2, \mathrm{t}_{\mathrm{o}}+\beta\right]\right)\right), 0=\beta=\mathrm{w} / 2\right\}\right) \\
& +0.5 \bullet \inf \left(\left\{\sup \left(\mathrm{f}\left(\left[\mathrm{t}_{\mathrm{o}}+\beta-\mathrm{w} / 2, \mathrm{t}_{\mathrm{o}}+\beta\right]\right)\right), 0=\beta=\mathrm{w} / 2\right\}\right)
\end{aligned}
$$

\subsection{Average Filter}

Before developing the continuous time definition of the median filter, it is helpful to examine the definition of the continuous time average, or mean, filter. In discrete time, the output of this filter is simply the mean of all samples in the filter window. In continuous time, acting on a signal $f(t)$ with a window of length w centered at $t_{0}$, the output of the average filter is:

$$
\operatorname{MEAN}_{\mathrm{ct}}\left\{\mathrm{f}\left(\left[\mathrm{t}_{\mathrm{o}}-\mathrm{w} / 2, \mathrm{t}_{\mathrm{o}}+\mathrm{w} / 2\right]\right)\right\}=\frac{1}{\mathrm{w}} \int_{\mathrm{t}_{\mathrm{o}} \mathrm{w} / 2}^{\mathrm{t}_{\mathrm{o}}+\mathrm{w} / 2} \mathrm{f}(\mathrm{t}) \mathrm{dt}
$$

Letting $\mathrm{a}\left(\mathrm{t}_{\mathrm{o}}\right)$ denote the average-filtered value of $\mathrm{f}(\mathrm{t})$ at $\mathrm{t}=\mathrm{t}_{\mathrm{o}}$, the above expression may be rearranged to yield a form which defines the continuous time median:

$$
\int_{t_{0}-w / 2}^{t_{o}+w / 2}\left[f(t)-a\left(t_{0}\right)\right] d t=0
$$


This equation achieves a balance of area of the function above and below its mean $\mathrm{a}\left(\mathrm{t}_{\mathrm{o}}\right)$ over the window. In contrast, the median achieves a balance of the length (or number of values) of the function above and below its value in the filter window.

\subsection{Median Filter}

One way to achieve the balance of values above and below the median is to apply the signum function to the difference between the input signal and proposed median value. The signum function returns a value of +1 for arguments greater than zero, 0 for arguments equal to zero, and -1 for arguments less than zero. Thus all values of the input greater than the median are assigned +1 , and all values less than the median are assigned -1 . These two areas should cancel out, just as in the preceding expression for the average filter. Letting $m\left(t_{o}\right)$ denote the median-filtered value of $\mathrm{f}(\mathrm{t})$ at $\mathrm{t}=\mathrm{t}_{\mathrm{o}}$, we have:

$$
\int_{t_{0}-w / 2}^{t_{o}+w / 2} \operatorname{sgn}\left\{f(t)-m\left(t_{0}\right)\right\} d t=0
$$

However, this definition is not valid when the input signal $\mathrm{f}(\mathrm{t})$ takes on the median value an infinite number of times inside the window $t_{0}-w / 2=t=t_{0}+w / 2$. When this is true, there will sometimes exist no value of $m\left(t_{0}\right)$ for which the above equality holds. In this case, the value of the integral will jump from a value less than zero for $\mathrm{m}\left(\mathrm{t}_{\mathrm{o}}\right)$ less than a certain value $\mathrm{m}_{\mathrm{o}}$, to a value greater than zero for $\mathrm{m}\left(\mathrm{t}_{\mathrm{o}}\right)$ greater than $\mathrm{m}_{\mathrm{o}}$. The value $\mathrm{m}_{\mathrm{o}}$ is then the median of the signal in the window and may be considered to be the value of $\mathrm{m}\left(\mathrm{t}_{\mathrm{o}}\right)$ that minimizes the absolute value of the integral in the above expression. The median of the signal is thus the value $m\left(t_{0}\right)$ that minimizes the expression:

$$
\left|\int_{t_{0}-w / 2}^{t_{0}+w / 2} \operatorname{sgn}\left\{f(t)-m\left(t_{0}\right)\right\} d t\right|
$$

Another situation which may cause difficulty in computing the continuous time median is an input signal with jump discontinuities. For example, when a perfect edge from a constant value 0 to a constant value 1 is centered in the filter window, the above integral expression is equal to zero for all $0<\mathrm{m}\left(\mathrm{t}_{\mathrm{o}}\right)<1$. This situation is analogous to a discrete time median of an even-sized window with half the values equal to 0 and half the values equal to 1 . In the discrete time case, the median is usually taken to be the average of the two values spanning the median. This definiton may be extended to continuous time simply by averaging the two values of $m\left(t_{0}\right)$ which limit from above and below the values for which the integral expression is zero. For the periodic signals used in this paper as input, such inderminate values occur only at isolated points and the specific handling of this situation does not affect the results.

Fitch, Coyle, and Gallagher ${ }^{4}$ described an "analog median filter" in 1986 which is identical to the continuous time median filter described in this paper. Using a different derivation than that given here, they give two distinct definitions which give the upper and lower limits in the indeterminate situation described above, and resolve the difference in favor of the upper limit. Their paper also formalizes the relationship between the discrete time and continuous time filters. 


\subsection{Midrange Filter}

The midrange filter outputs the average of the minimum and maximum values in the filter window. This filter generalizes very easily to continuous time by using the infimum and supremum. The midrange filter output, $\mathrm{MIDR}_{\mathrm{ct}}$, of an input signal $\mathrm{f}(\mathrm{t})$ at $\mathrm{t}=\mathrm{t}_{\mathrm{o}}$ for window size $\mathrm{w}$ is:

$$
\operatorname{MIDR}_{\mathrm{ct}}\left\{\mathrm{f}\left(\left[\mathrm{t}_{\mathrm{o}}-\mathrm{w} / 2, \mathrm{t}_{\mathrm{o}}+\mathrm{w} / 2\right]\right)\right\}=0.5 \cdot \sup \left\{\mathrm{f}\left(\left[\mathrm{t}_{\mathrm{o}}-\mathrm{w} / 2, \mathrm{t}_{\mathrm{o}}+\mathrm{w} / 2\right]\right)\right\}+0.5 \bullet \inf \left\{\mathrm{f}\left(\left[\mathrm{t}_{\mathrm{o}}-\mathrm{w} / 2, \mathrm{t}_{\mathrm{o}}+\mathrm{w} / 2\right]\right)\right\}
$$

The midrange filter is neither good at suppressing impulses nor preserving edges, but yields good results on signals corrupted by uniformly-distributed noise. Because of it is the average of two extremum operations, it resembles in some situations the pseudomedian filter. In fact, the midrange filter is the average of the morphological erosion and dilation of a signal or image.

\section{RESPONSE TO PERIODIC SIGNALS}

\subsection{Assumptions}

In this section we derive a type of "response curve" for the four filters under consideration to three periodic waves: triangle waves, sinusoidal waves, and square waves. Several assumptions underlying the response curves need to be clarified before proceeding, however.

First, the reponse curves developed here do not give the filter response to a combination of frequencies, but instead only to a single frequency of a particular periodic signal. The curves are derived by determining how the response of the filter changes as the frequency of a single periodic signal is varied. The superposition principle, used extensively for linear filters, does not apply to the nonlinear filters in this study.

Second, "response" is used here only to denote amplitude and phase of the output as compared to the input. Any distortion induced by the filter is not considered in the response analysis; the distortion analysis using a correlation measure provides information on these aspects of the filter behavior. The "amplitude response" values in this paper give the ratio of the peak values of the filtered signal to the peak values of the unfiltered signal. Many of the filtered signals exhibit shape distortion, especially median-filtered signals and all filtered signals with very small amplitudes. The filtered signals always have the same basic period as the input signals, however. The "phase response" is assumed to be the phase relationship between filtered and unfiltered signal peaks; under this assumption, the phase of the filtered signal is always 0 or $180^{\circ}$. To simplify the presentation of the data, we represent a filtered signal $180^{\circ}$ out of phase with the original as having a negative amplitude response, which is the practical effect of this phase shift.

Third, each filter responds differently to different types of periodic signals. This is clearly because the filters are nonlinear, but is also due in small part to the assumptions used to define "response." The results indicate similarities in a filter's response to many types of periodic signals that reveal some general characteristics of the filter, however.

Finally, the response of actual discrete time filters varies significantly from the responses shown in this section. These differences arise not only from assumptions inherent in converting discrete time filters to continuous time but also from sampling and quantization effects. 


\subsection{Response to Triangle Waves}

One input signal for which it is easy to determine the peak output values of the various filters is the triangle wave. In fact, the response may be represented in a closed form as a function of the input signal frequency. For the pseudomedian filter, the ratio of the peaks of the filtered and unfiltered signals will be represented by $\mathrm{P}_{\mathrm{tr}}(\mathrm{f})$, where $\mathrm{f}$ is the frequency of the signal given in units of cycles per filter window length, which will be abbreviated $\mathrm{cy} / \mathrm{w}$.

$$
\begin{array}{cc}
\mathrm{P}_{\mathrm{tr}}(\mathrm{f})=1-\mathrm{f} / 2, & 0=\mathrm{f}=2 \mathrm{cy} / \mathrm{w} \\
0, & \mathrm{f}>2 \mathrm{cy} / \mathrm{w}
\end{array}
$$

The response of the median filter to triangle waves, $\mathrm{M}_{\mathrm{tr}}(\mathrm{f})$, is quite different:

$$
\begin{aligned}
& \mathrm{M}_{\mathrm{tr}}(\mathrm{f})=1-\mathrm{f}, \quad 0=\mathrm{f}=1 \frac{1 / 3}{\mathrm{cy} / \mathrm{w}} \\
& \mathrm{f} / 2-1, \quad 1 \frac{1 / 3}{\mathrm{cy} / \mathrm{w}}<\mathrm{f}=22 / 5 \mathrm{cy} / \mathrm{w} \\
& 1-\mathrm{f} / 3, \quad 22 / 5 \mathrm{cy} / \mathrm{w}<\mathrm{f}=33 / 7^{\mathrm{cy} /}{ }_{\mathrm{w}} \\
& \text {... etc. }
\end{aligned}
$$

Or, more formally,

$$
\begin{aligned}
M_{\mathrm{tr}}(\mathrm{f})= & 1-\frac{\mathrm{f}}{2 \mathrm{n}+1}, \quad\left(2 \mathrm{n}+\frac{2 \mathrm{n}}{4 \mathrm{n}+1}\right) \mathrm{cy} / \mathrm{w} \leq \mathrm{f} \leq\left(2 \mathrm{n}+1+\frac{2 \mathrm{n}+1}{4 \mathrm{n}+3}\right) \mathrm{cy} / \mathrm{w} \\
& \frac{\mathrm{f}}{2 \mathrm{n}+2}-1, \quad\left(2 \mathrm{n}+1+\frac{2 \mathrm{n}}{4 \mathrm{n}+3}\right) \mathrm{cy} / \mathrm{w} \leq \mathrm{f} \leq\left(2 \mathrm{n}+2+\frac{2 \mathrm{n}+2}{4 n+5}\right) \mathrm{cy} / \mathrm{w}
\end{aligned}
$$

where $\mathrm{n}=0,1,2,3, \ldots$

These response curves are illustrated in Figure 1 below.

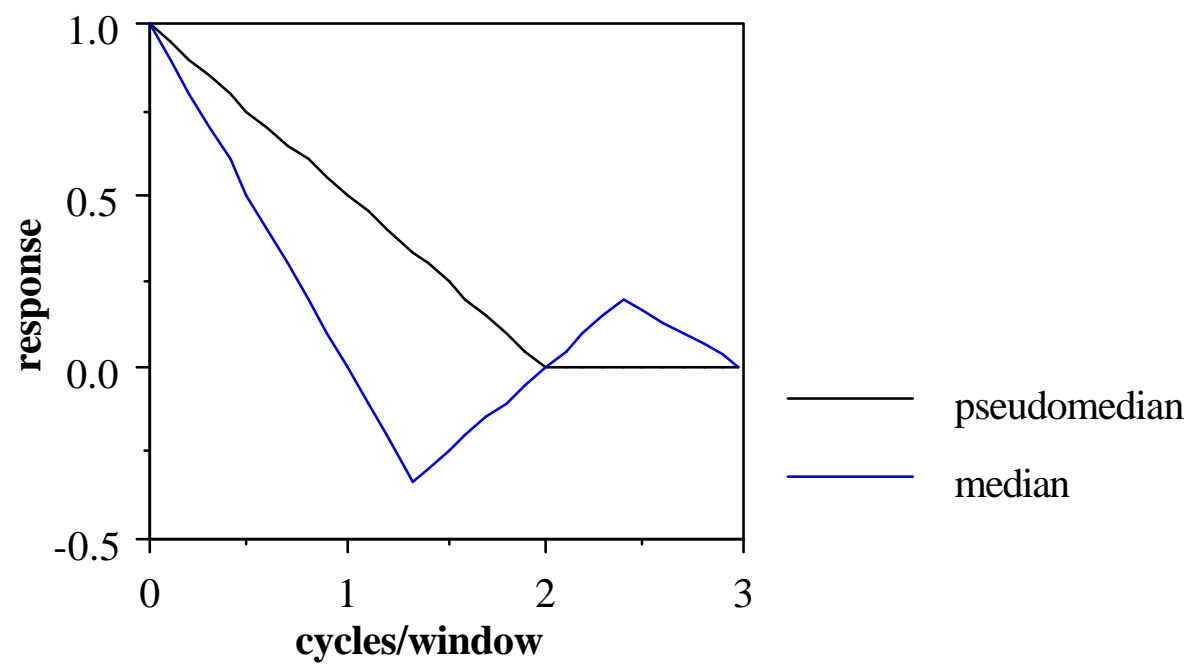

Figure 1. Response of pseudomedian and median filters to triangle waves.

Similar response curves derived for the average and midrange filters offer comparisons between these filters and the median and pseudomedian filters. The response of the average filter to triangle waves, $A_{t r}(f)$, is:

$$
\begin{aligned}
\mathrm{A}_{\mathrm{tr}}(\mathrm{f})= & (-1)^{\mathrm{n}}[-\mathrm{f}+(2 \mathrm{n}+1)-\mathrm{n}(\mathrm{n}+1) / \mathrm{f}], \quad \mathrm{n} \text { cy/w }=\mathrm{f}=(\mathrm{n}+1) \mathrm{cy} / \mathrm{w} \\
& \text { where } \mathrm{n}=0,1,2,3, \ldots
\end{aligned}
$$


The response of the midrange filter to triangle waves, $R_{t r}(f)$, is simply:

$$
\begin{array}{cc}
\mathrm{R}_{\mathrm{tr}}(\mathrm{f})=1-\mathrm{f}, & 0=\mathrm{f}=1 \mathrm{cy} / \mathrm{w} \\
0, & \mathrm{f}>1 \mathrm{cy} / \mathrm{w}
\end{array}
$$

These response curves are illustrated in Figure 2 below.

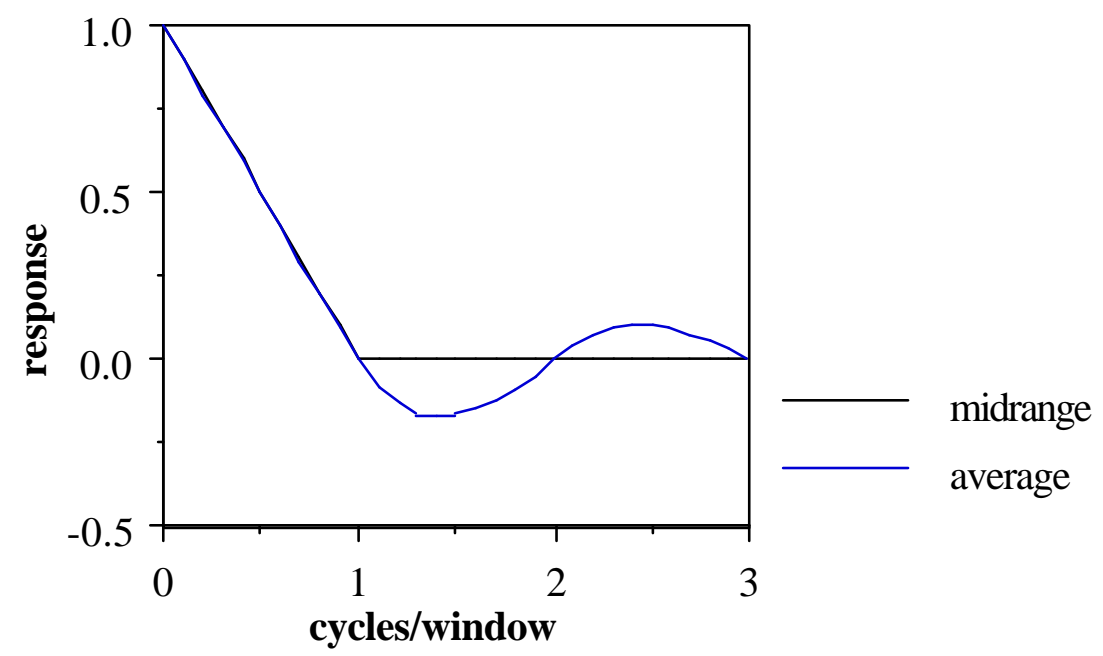

Figure 2. Response of midrange and average filters to triangle waves.

A comparison of Figures 1 and 2 demonstrates that the median and average filters have similar responses to triangle waves, while pseudomedian and midrange filters also have closely related responses. In fact, a midrange filter has the identical peak response to a pseudomedian filter with a window twice as wide as that of the midrange filter. This fact is related to the effective window size change created by sequential erosion and dilation when computing the morphological open and close $^{3}$.

\subsection{Sinusoidal Waves}

Sinusoidal waves resemble triangle waves in many respects, and therefore one would expect the response curves of the filters to sinusoidal waves to be similar the curves for triangle waves. Indeed, the most obvious difference between the response curves is sinusoidal curves introduced where there were previously straight lines. The pseudomedian filter response to sinusoidal waves, $\mathrm{P}_{\mathrm{sn}}(\mathrm{f})$, is given by:

$$
\begin{array}{cc}
\mathrm{P}_{\mathrm{sn}}(\mathrm{f})=1 / 2(1+\cos \mathrm{pf} / 2), & 0=\mathrm{f}=2 \mathrm{cy} / \mathrm{w} \\
0, & \mathrm{f}>2 \mathrm{cy} / \mathrm{w}
\end{array}
$$

For the median filter acting on sinusoidal waves, the response $\mathrm{M}_{\mathrm{sn}}(\mathrm{f})$ is:

$$
\begin{array}{ccc}
\mathrm{M}_{\mathrm{sn}}(\mathrm{f})= & \cos (\mathrm{pf} / 2), & 0=\mathrm{f}=11 / 3 \mathrm{cy} / \mathrm{w}^{2} \\
-\cos (\mathrm{pf} / 4), & 11 / 3 \mathrm{cy} /{ }_{\mathrm{w}}<\mathrm{f}=22 / 5 \mathrm{cy} / \mathrm{w} \\
\cos (\mathrm{pf} / 6), & 22 / 5 \mathrm{cy} /{ }_{\mathrm{w}}<\mathrm{f}=33 / 7 \mathrm{cy} / \mathrm{w} \\
\ldots & \text { etc. }
\end{array}
$$

Or, more formally, 


$$
\begin{aligned}
M_{\text {sn }}(f)= & \cos \left(\frac{\pi f}{4 n+2}\right), \quad\left(2 n+\frac{2 n}{4 n+1}\right) c y / w \leq f \leq\left(2 n+1+\frac{2 n+1}{4 n+3}\right) c y / w \\
& -\cos \left(\frac{\pi f}{4 n+4}\right), \quad\left(2 n+1+\frac{2 n}{4 n+3}\right) c y / w \leq f \leq\left(2 n+2+\frac{2 n+2}{4 n+5}\right) c y / w
\end{aligned}
$$

These curves are plotted in Figure 3 below.

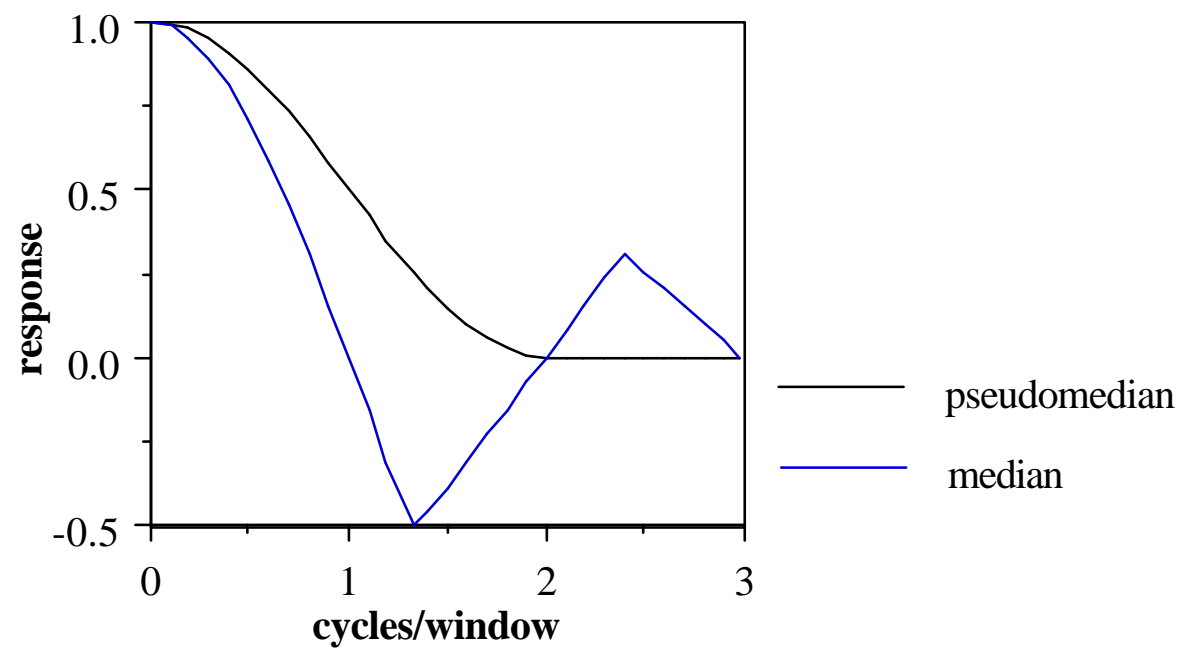

Figure 3. Response of pseudomedian and median filters to sinusoidal waves.

Figures 1 and 3 show the similarities in these filters' responses to triangle and sinusoidal waves. To complete the comparison with the average and midrange filters, the expressions for these filters' responses to sinusoidal waves are given below and plotted in Figure 4. $\mathrm{A}_{\mathrm{sn}}(\mathrm{f})$ is the response of the average filter to sinusoidal waves and $R_{\mathrm{sn}}(\mathrm{f})$ is the response of the midrange filter to sinusoidal waves.

$$
\begin{aligned}
& \mathrm{A}_{\mathrm{sn}}(\mathrm{f})=\operatorname{sinc} \mathrm{pf}, \quad \mathrm{f}=0 \\
& R_{\mathrm{sn}}(\mathrm{f})=1 / 2(1+\cos p f), \quad 0=\mathrm{f}=1 \mathrm{cy} / \mathrm{w} \\
& 0, \quad \mathrm{f}>1 \mathrm{cy} / \mathrm{w}
\end{aligned}
$$

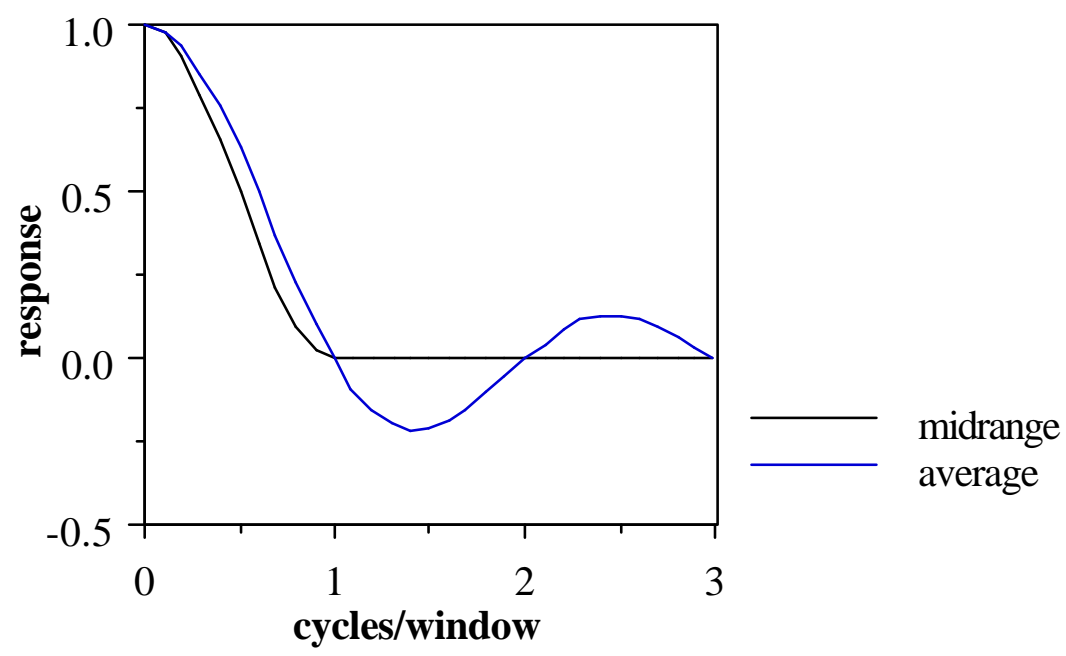

Figure 4. Response of average and midrange filters to sinusoidal waves. 
The above results show some very significant similarities and differences between the filters. The pseudomedian filter has a uniformly decreasing response from unity to zero to sinusoidal and triangular waves as the frequency increases from zero to two cycles per window. At frequencies above two cycles per window, the pseudomedian filter always gives zero output (in continuous time) for triangle and sinusoidal waves. More generally, the pseudomedian filter reduces any vertically symmetric periodic signal of frequency greater than or equal two cycles per window to a constant output equal to the point of symmetry. The midrange filter gives a similar result, except that it produces a constant output for all frequencies at and above one cycle per window. The median filter, however, does not block high frequency signals, and in fact exhibits phase inversion $\left(180^{\circ}\right.$ phase shift) for frequencies between one and two cycles per window (and also for 3-4 cy/w, $5-6 \mathrm{cy} / \mathrm{w}$, and so forth). The average filter has responses similar to those of the median filter for triangle and sinusoidal waves, including phase inversion in the same frequency ranges. These similarities are understandable since both the median and average filters are inherently low-pass and these examples do not demonstrate the edge-preserving or impulse-removing abilities of the median filter.

\subsection{Square Waves}

The response of the filters to square waves is easy to analyze. The median filter may only have two distinct output values, since there are only two different input values for a square wave, and the pseudomedian and midrange filters may have only three distinct output values. The average filter, however, is not similarly limited in its outputs. The responses of the four filters to square waves are given below.

$$
\begin{aligned}
& \text { Pseudomedian: } P_{\mathrm{sq}}(\mathrm{f})=1, \quad 0=\mathrm{f}<1 \mathrm{cy} / \mathrm{w} \\
& 0, \quad \mathrm{f}>1 \mathrm{cy} / \mathrm{w}
\end{aligned}
$$

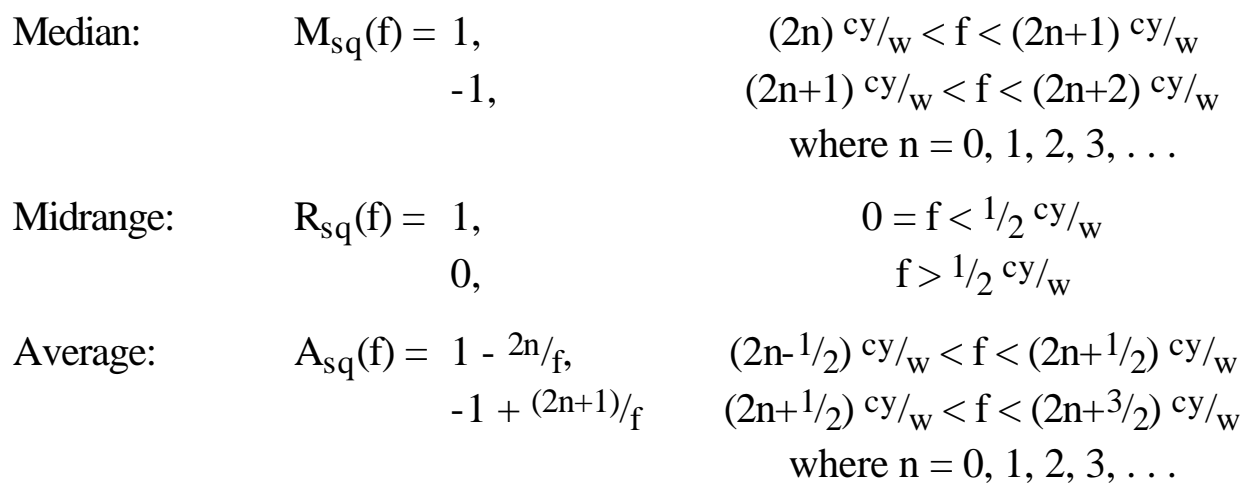

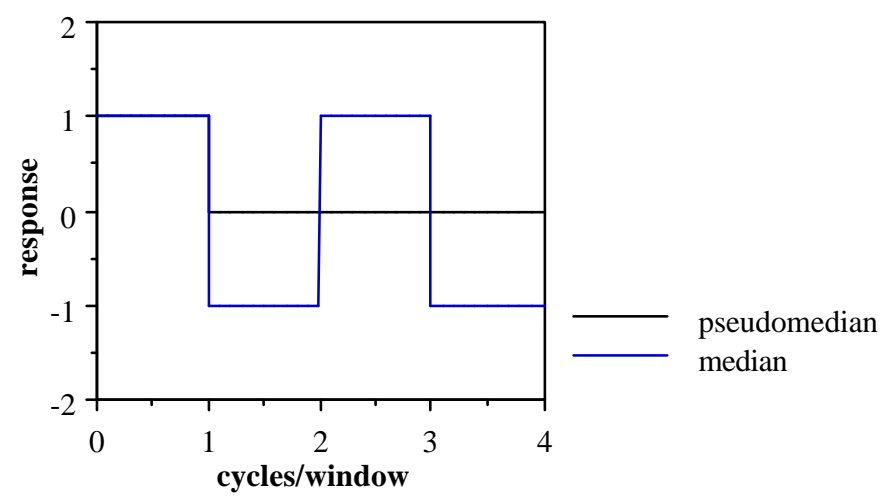

Figure 5. Response of pseudomedian and median filters to square waves. 


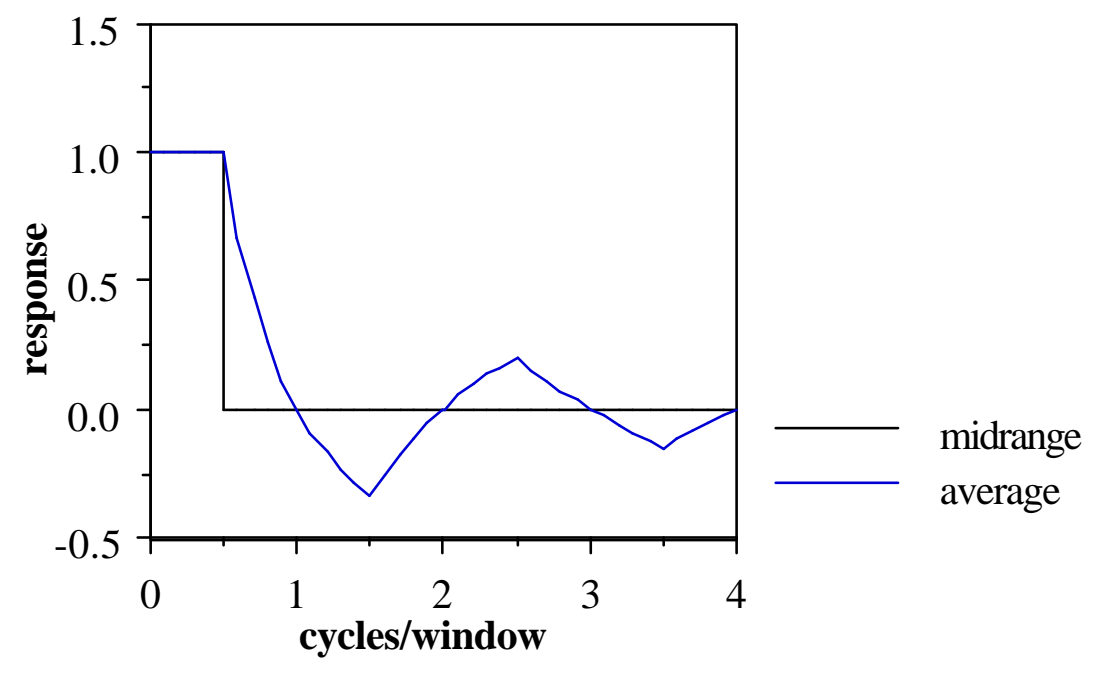

Figure 6. Response of midrange and average filters to square waves.

Once again, the midrange and pseudomedian filter have responses differing by a factor of two in frequency. The median and average filters, however, respond quite differently to square waves. They both still exhibit phase inversion, but the median filter does not attenuate square waves at all. This is because square waves are bi-valued and therefore are either infinite-length roots or oscillatory roots of the median filter 5 . In contrast, the output of the pseudomedian filter for fast-fluctuating (greater than $2 \mathrm{cy} / \mathrm{w}$ ) bi-valued signals is a constant value.

The relationship between the continuous time and discrete time median filters has been formalized by Fitch, Coyle, and Gallagher ${ }^{4}$. The relationship between the other continuous and discrete filters has not been demonstrated, but results given by Schulze ${ }^{6}$ show excellent agreement between the continuous time filters and discrete time filters with large window sizes. Discrete filters with smaller window sizes show significant variance from the continuous time results, but most of the differences can be easily explained by sampling, quantization, and windowing effects. In any case, the general properties of the filters demonstrated in continuous time are generally valid in discrete time as well.

\section{DISTORTION ANALYSIS}

The response curves given in the preceding section are only a comparison of the amplitudes of the input and output signals and provide no information about how extensively the filters modify the shapes of the waveforms. To supplement the amplitude information with information about the distortion induced by the filters, we investigated the use of the statistical correlation between the input and output. However, the correlation is very sensitive to amplitude and phase differences between the signals, so a correlation taken directly between the input and output yields much the same result as the above response curves. To restrict the measure only to the change in shape of the waveform, the output signal is adjusted to compensate for changes in the amplitude and phase of the signal. The resulting correlation measure is restricted to the range 0 to 1 , since negative correlation indicates a phase shift. Correlation equal to +1 indicates exact correspondence between signals, while correlation equal to 0 indicates no correspondence at all, as between a periodic signal and its DC component.

Consider a continuous input signal $\mathrm{x}(\mathrm{t})$ and the corresponding filter output $\mathrm{y}(\mathrm{t})$, both with period $\mathrm{T}$. Define the gain of the filter operating on the signal $x(t)$ to be: 


$$
\operatorname{GAIN}=\frac{\left(\int_{k}^{\mathrm{k}+\mathrm{T}}[y(t)]^{2} \mathrm{dt}\right)^{1 / 2}}{\left(\int_{\mathrm{k}}^{\mathrm{k}+\mathrm{T}}[\mathrm{x}(\mathrm{t})]^{2} \mathrm{dt}\right)^{1 / 2}}
$$

where $\mathrm{k}$ is an arbitrary constant. For frequencies where the output signal is $180^{\circ}$ out of phase with the input signal, this GAIN figure is negated to adjust for the phase shift. This is only valid for vertically symmetric periodic signals such as those considered in this paper. The correlation figure is then:

$$
\operatorname{CORR}=1-\frac{\left(\int_{k}^{\mathrm{k}+\mathrm{T}}\left|\mathrm{x}(\mathrm{t})-\frac{1}{\operatorname{GAIN}} \mathrm{y}(\mathrm{t})\right|^{2} \mathrm{dt}\right)^{1 / 2}}{\left(\int_{\mathrm{k}}^{\mathrm{k}+\mathrm{T}}[\mathrm{x}(\mathrm{t})]^{2} \mathrm{dt}\right)^{1 / 2}}
$$

Unfortunately, deriving expressions for the gain and correlation in continuous time is extremely difficult, since the value of the output must be computed at all times for all frequencies for each signal and filter, instead of merely deducing the peak amplitude of the output. To give some indication of how the four filters distort the signals considered in this paper, we have computed the gain and correlation digitally (using summations over one period instead of integrals) for 75-wide filters. The triangle, sinusoidal, and square wave signals were considered at frequencies from 0 to $4 \mathrm{cy} / \mathrm{w}$ in increments of $0.05 \mathrm{cy} / \mathrm{w}$. The results are shown in Figures 7, 8, and 9 below. We chose very wide filter windows to minimize the discrete time effects. These results show that the pseudomedian filter has very high correlation (that is, very low distortion) throughout most of its passband for all three input signals. The median filter distorts the triangle and sinusoidal waves much more than the square wave, which is expected from its edge preserving and impulse removing abilities. The average filter is the best of the four filters at preserving the sinusoidal waveform, which is understandable because this filter smooths signals to create only gradual changes, much like those in the original sinusoid. The midrange filter causes particularly high distortion on the square wave because it destroys edges. The distortion analysis shows that the pseudomedian filter distorts these input signals in its passband less than any of the other filters except for the average filter on sinusoidal waves.

\section{CONCLUSIONS}

The continuous time filter analysis demonstrates the similarities and differences among the pseudomedian, median, average, and midrange filters. Comparing only the peak amplitude response of the filters to triangle, sinusoidal, and square waves highlights relationships between the pseudomedian and midrange filters and between the median and average filters. Distortion analysis, however, highlights the differences in responses between the edge-preserving filters (median and pseudomedian) and the edge-destroying filters (average and midrange). The pseudomedian and midrange filters are shown to completely attenuate any symmetric periodic signal above a 
certain frequency ( 2 and 1 cycles per window length, respectively), while the median and average filters always give some periodic, often phase-inverted, output to such high-frequency inputs. In addition, the pseudomedian filter distorts periodic signals much less in most cases than the other filters.

Although the continuous time analysis of the filters cannot be directly applied to filters operating in discrete time, the general behavior of the filters relative to one another remains the same in the discrete case. For example, the response of the median filter to square waves clearly shows why infinite-length fast-fluctuating bi-valued roots and oscillating roots exist for this filter in discrete time. The response of the pseudomedian filter to square waves in continuous time indicates that it does not have any such fast-fluctuating roots, and indeed this is the case ${ }^{6}$. This paper has also focused exclusively on the filters in one dimension, so direct application of these results to two dimensions is not possible. Again, though, the general behavior the filters demonstrate in this one-dimensional analysis has implications for their two-dimensional behavior, especially with respect to their susceptibility to highfrequency noise and the amount of distortion they induce in an image.

\section{ACKNOWLEDGMENTS}

This material is based upon work supported under a National Science Foundation Graduate Fellowship.

\section{REFERENCES}

1. W. K. Pratt, T. J. Cooper, and I. Kabir. "Pseudomedian filter." Proc SPIE, v. 534 (1985) pp. 34-43.

2. W. K. Pratt. Digital Image Processing, 2nd edition. New York: Wiley, 1991.

3. M. A. Schulze and J. A. Pearce. "The pseudomedian and LOCO filters: linear combinations of morphological operators." Submitted to SPIE Conference on Image Algebra and Morphological Image Processing III, San Diego, July 1992.

4. J. P. Fitch, E. J. Coyle, and N. C. Gallagher. "The analog median filter." IEEE Trans Circuits and Syst, v. CAS-33 n. 1 (1986) pp. 94-102.

5. S. G. Tyan. "Median filtering: deterministic properties." Two-Dimensional Signal Processing II, T. S. Huang, ed. Berlin: Springer-Verlag, 1981. pp. 197-217.

6. M. A. Schulze. "Mathematical properties of the pseudomedian filter." M.S. thesis, University of Texas at Austin, 1990.

7. M. A. Schulze and J. A. Pearce. "Some properties of the two-dimensional pseudomedian filter." Proc SPIE, v. 1451 (1991) pp. 48-57.

8. P. Maragos and R. W. Schafer. "Morphological filters" Parts I and II. IEEE Trans Acoust Speech Signal Process, v. ASSP-35 n. 8 (1987) pp. 1153-1184. 


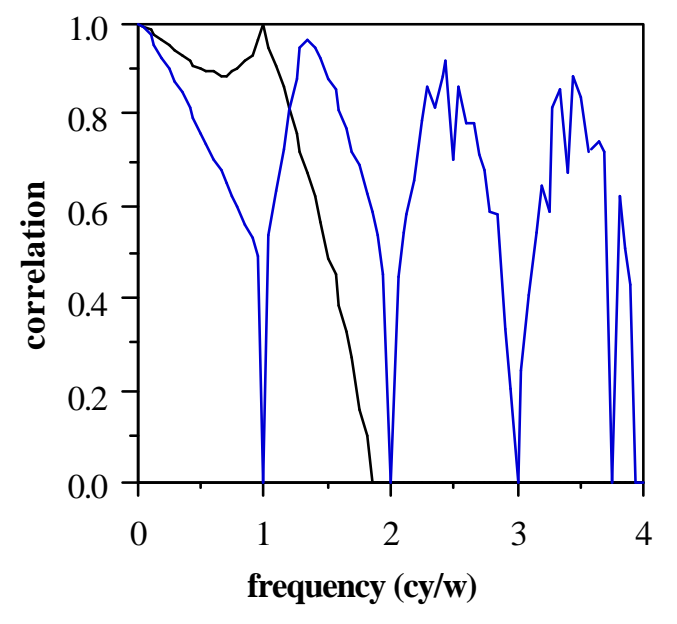

pseudomedian

Figure 7. Correlation curves for 75-wide filters acting on triangle waves.
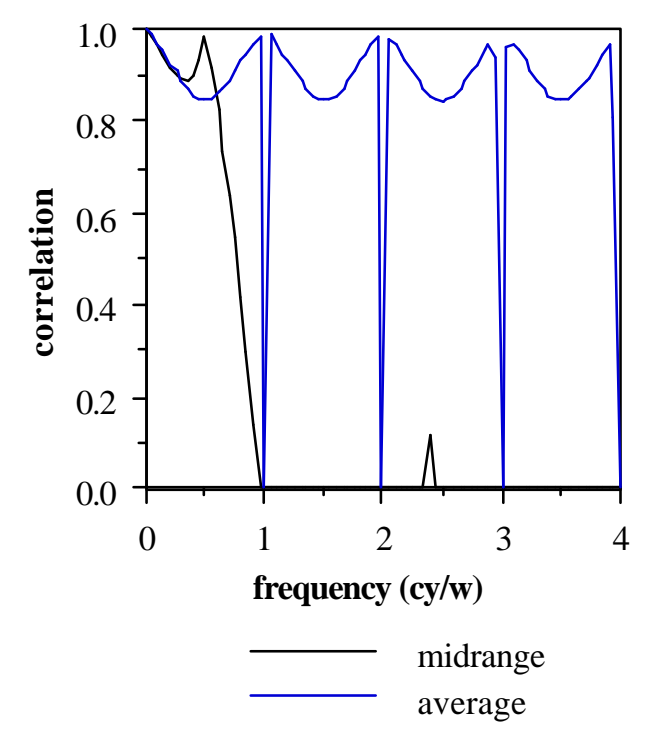
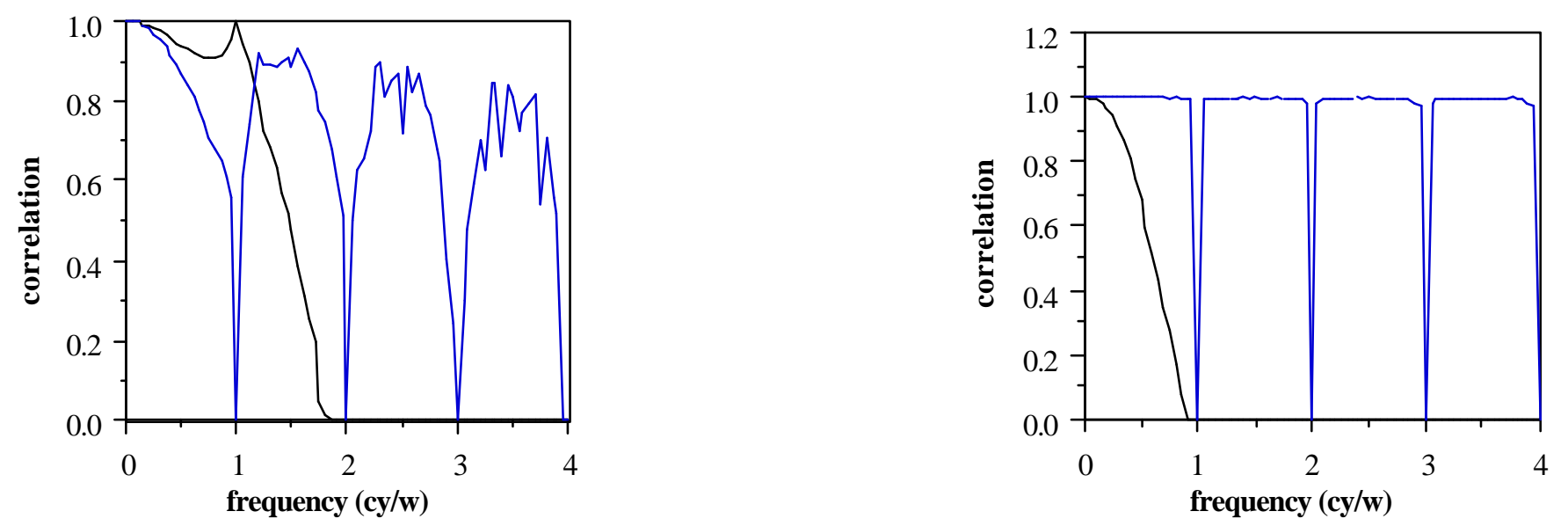

Figure 8. Correlation curves for 75-wide filters acting on sinusoidal waves. (Legends as in Fig. 7.)
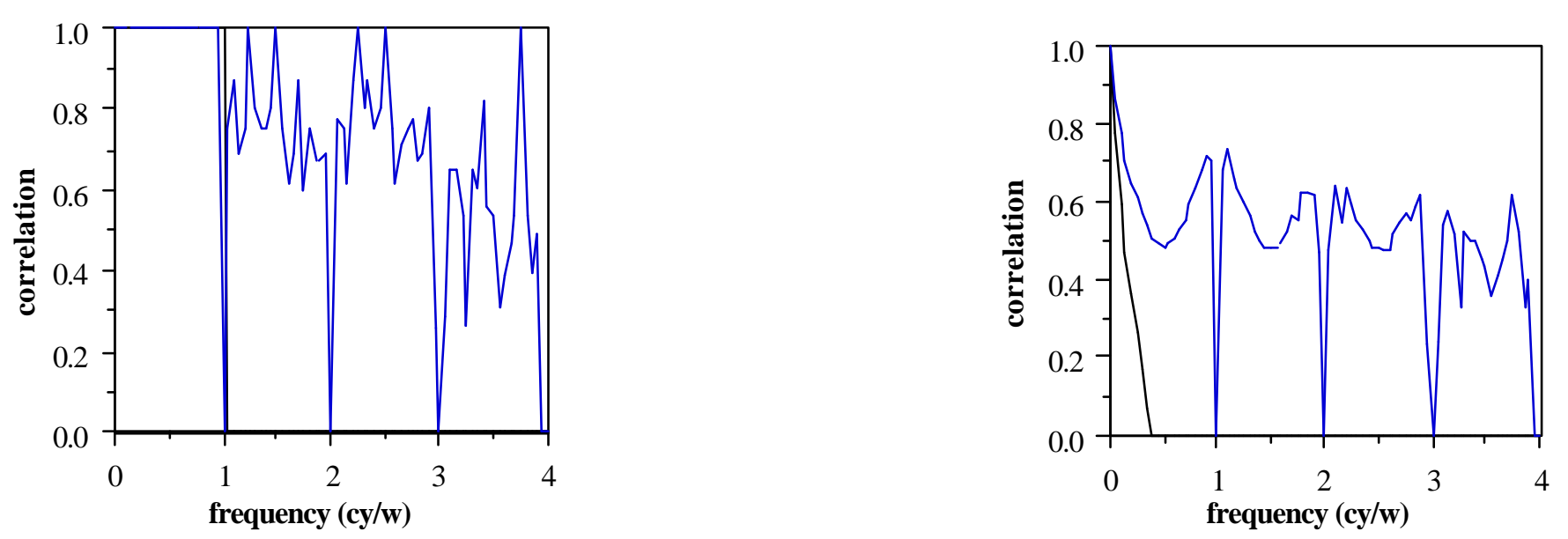

Figure 9. Correlation curves for 75-wide filters acting on square waves. (Legends as in Fig. 7.) 